\title{
Caracterização de rochas areno-argilosas da Formação Maracangalha por RMN
}

Nathália de Souza Penna* e Joelson da C. Batista (*USP, UFBA)

Copyright 2021, SBGf - Sociedade Brasileira de Geofísica.

This paper was prepared for presentation at the $17^{\text {th }}$ International Congress of the Brazilian Geophysical Society, held in Rio de Janeiro, Brazil, August 16-19, 2021

Contents of this paper were reviewed by the Technical Committee of the $17^{\text {th }}$ International Congress of The Brazilian Geophysical Society and do not necessarily represent any position of the SBGf, its officers or members. Electronic reproduction or storage of any part of this paper for commercial purposes without the written consent of The Brazilian Geophysical Society is prohibited.

\section{Abstract}

Geophysical prospecting of hydrocarbon reservoirs includes various techniques and different geophysical methods. Since drilling and logging of wells may prove to be a costly endeavor, the results provided by surface geophysical research methods are used to assist in determining regions where such investments are warranted. Among the methods used in the preliminary stages of geological and geophysical characterization of a given prospectus is the laboratory study of the petrophysical properties of outcrops that have similar textures and/or rock structures to those that make up the so-called petroleum system. From the analysis of properties such as porosities, permeability, saturation, capillary pressure and clay mineral content it is possible to infer the potential of a given rock formation to be a good reservoir. In this context, the present work was developed to add to other petrophysical studies that seek to better understand how such properties associate to define exploitable reservoirs. In this research, from the analysis of 31 samples of rocks from outcrops of the region of Ilha dos Frades, Bahia, Brazil, the petrophysical properties of the Formação Maracangalha, one of the main hydrocarbon reservoirs of the Bacia do Recôncavo, were estimated and evaluated, using the Nuclear Magnetic Resonance (NMR) technique. Sandstones of different textures were characterized in terms of porosity, permeability, saturation and petrofacies analysis. The results obtained with NMR were compared to those provided by traditional laboratory methods.

\section{Introdução}

A capacidade de armazenamento e produção de reservatórios é função das propriedades das rochas e dos fluidos que as saturam. A viabilidade de um dado reservatório ser explorado economicamente pode ser determinada a partir da análise de certas características tais como: identificação das litologias que constitui a rocha, natureza dos fluidos que preenchem o espaço poroso, porosidade, permeabilidade, teor de saturação e argilosidade.

As ferramentas mais utilizadas para obtenção de parâmetros petrofísicos são baseadas em contrastes entre massas específicas de substâncias, propriedades elétricas, propriedades elásticas associadas à propagação de ondas mecânicas, ou ainda, em propriedades inerentes ao átomo que determinam as interações envolvendo partículas subatômicas (nêutrons e elétrons). No entanto, a maioria dessas ferramentas apresentam limitações relacionadas à precisão dos dados observados: os valores obtidos com tais ferramentas tendem a refletir bastante os aspectos inerentes à técnica empregada, à geometria da ferramenta e às condições ambientais.

As ferramentas pautadas no fenômeno da RMN foram desenvolvidas no final do século $X X$ e ganharam destaque por serem capazes de fornecer valores mais acurados de porosidades do que a maioria das chamadas técnicas tradicionais. Alguns estudos têm tornado as medições de RMN uma rotina em muitas aplicações petrofísicas, principalmente aquelas relacionadas a rochas siliciclásticas (Kenyon 1997; Martinez and Davis, 2000). A aplicação da RMN apresenta como principais vantagens os seguintes aspectos: 1) os valores de porosidade observados são função das propriedades do fluido e do espaço poroso e assim, diferente das técnicas tradicionais, a matriz da rocha não exerce influência substancial sobre o parâmetro físico medido; 2) comparada a outras ferramentas utilizadas em laboratório, a aplicação da RMN favorece a preservação das amostras, uma vez que, consiste em um processo não-destrutivo e 3) como predominantemente a interação entre fluidos e volume poroso afetam os dados medidos, os resultados adquiridos em laboratório podem ser reproduzidos em quaisquer ambientes, inclusive em campo. Nesse trabalho, a técnica de RMN foi empregada em medidas laboratóriais em amostras de afloramentos da Formação Maracangalha, Bacia do Recôncavo, Bahia, Brasil, com os seguintes objetivos: 1)caracterizar em termos de porosidade, índice de fluido livre, índice de fluido ligado e permeabilidade, os arenitos com petrofácies distintas dessa formação; e 2) analisar os resultados obtidos com a finalidade de fornecer uma descrição acerca das propriedades petrofísicas que definem tais arenitos.

\section{Geologia da Área de Estudo}

A Bacia do Recôncavo localiza-se no Nordeste do Brasil, no centro-leste da Bahia e compreende uma área de aproximadamente $11.500 \mathrm{~km}^{2}$. Essa bacia é limitada a norte e noroeste com o Alto de Aporá, que a separa da Bacia do Tucano Sul; ao oeste pela falha de Maragogipe; ao sul pelo sistema de falhas da Barra, que a separa da Bacia de Camamu e a leste pelo sistema de falhas de Salvador (Figura 1). Esta bacia constitui um segmento de rifte intracontinental abortado, cuja origem está associada ao processo de estiramento crustal que culminou na fragmentação do paleocontinente Gondwana e abertura do Oceano Atlântico Sul, durante o Eocretáceo.

Divida em dois membros e caracterizada por depósitos 


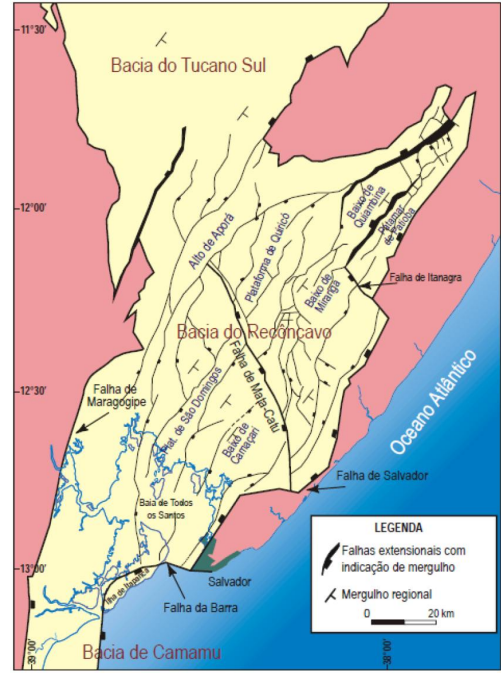

Figure 1: Localização e arcabouço estrutural da Bacia do Recôncavo. Fonte: Milhomem et al. (2003).

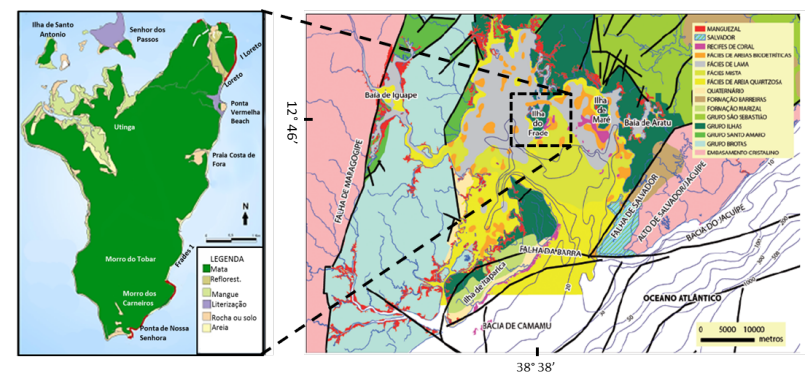

Figure 2: Localização da llha dos Frades e dos afloramentos. Modificado de Magnavita et al.(2005), Bittencourt et al. (1976) e Medeiros (2008).

relacionados a fluxos gravitacionais, a Formação Maracangalha consiste em um dos principais reservatórios da Bacia do Recôncavo, destacando-se pelos significativos volumes de gás armazenados nos arenitos intercalados com folhelhos lacustres (Milhomem et al., 2003). O seu membro Pitanga é caracterizado pela presença de arenitos mais argilosos e fácies com maior grau de deformação. Já o membro Caruaçu é composto por arenitos de granulometria fina a média, com estratificações plano-paralelas e cruzadas tangenciais, que teriam sido depositados a partir de deslizamento, escorregamento e/ou fluxos turbidíticos (Caixeta,1988; Magalhaes, 1990).

As amostras de arenitos utilizadas nessa pesquisa foram oriundas dos afloramentos da Formação Maracangalha nas localidades de Loreto e Ponta de Nossa Senhora na llha dos Frades, Bahia (Figura 2). As amostras analisadas oriundas do afloramento na localidade de Loreto receberam o prefixo LOR e compreendem arenitos com granulometria predominantemente fina e abundante fragmentos vegetais. Já as amostras de Ponta de Nossa Senhora, prefixo PNS, consistem em arenitos de granulometria fina, argilosos e de coloração acinzentada (Figura 3).

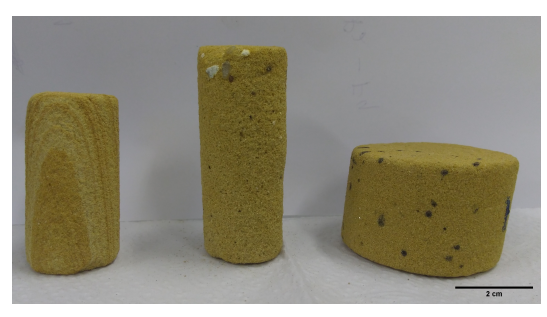

(a) LOR.

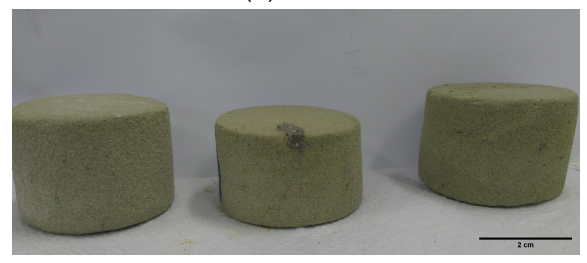

(b) PNS.

Figure 3: Amostras de rochas a) LOR e b)PNS.

\section{Metodologia}

Os parâmetros petrofísicos analisados a partir da utilização de técnicas de Ressonância Magnética Nuclear (RMN) nessa pesquisa foram: porosidade, índice de fluido livre (FFI), índice de fluido ligado (BVI), saturação irredutível e permeabilidade. Uma metodologia tradicional para obtenção de porosidade também foi aplicada: utilização de Ultrapicnômetro.

\section{Preparação de amostras}

As amostras utilizadas foram oriundas de procedimentos de amostragens realizadas pela equipe do Laboratório de Petrofísica (LaPetro) da Universidade Federal da Bahia. Antes das análises laboratoriais, as amostras foram limpas. O processo de limpeza aplicado foi baseado na técnica de Dean-Stark, na qual o solvente é continuamente aquecido, condensado e destilado sobre as amostras. O solvente utilizado foi o Metanol (álcool metílico, $\mathrm{CH}_{3} \mathrm{OH}$ ), em decorrência da eficiência desse em dissolver sais. $O$ tempo médio no qual as amostras foram submetidas ao processo de limpeza a partir da destilação do solvente foi seis horas. Depois da limpeza, as amostras foram colocadas em um forno Thelco a $100^{\circ} \mathrm{C}$ com a finalidade de eliminar resíduos de metanol no espaço poroso a partir da evaporação.

Para obter dados de porosidade a partir do equipamento de RMN é necessário que as amostras estejam 100\% saturadas. Assim, as amostras foram colocadas em um recipiente com $3 / 4$ de seu volume preenchido com a solução saturante, de modo que, ficassem quase completamente imersas no fluido. Esse recipiente contendo amostras e solução saturante, foi colocado em um dessecador associado a uma bomba a vácuo. Com o objetivo de simular condições de salinidade análogas aos reservatórios de campos de petróleo, uma solução de 70.000 ppm de $\mathrm{NaCl}$ foi utilizada para saturar essas amostras.

As amostras também foram submetidas em condições de saturação irredutível para obter estimativas de permeabilidade. $O$ processo de dessaturação das amostras foi obtida com 0 uso do equipamento 
Soilmoisture CAT 1500, no qual as amostras saturadas foram submetidas à pressão de aproximadamente 8 bar, por um tempo médio de 96 horas.

\section{Ressonância Magnética Nuclear (RMN)}

O sinal da RMN resulta da interação entre o movimento de precessão dos momentos magnéticos nucleares e o campo externo aplicado. Somente átomos com número de prótons e/ou neutrôns ímpares podem viabilizar a observação do sinal associado à RMN. Como o isótopo $H_{1}$ possui abundância natural elevada, maior sensibilidade absoluta e constante giromagnética, a maioria das ferramentas de RMN são baseadas nas respostas dos núcleos dos átomos de hidrogênio.

A constante de relaxação magnética transversal $\left(T_{2}\right)$ é o parâmetro físico preferencialmente utilizado nas ferramentas de RMN para obter informações sobre porosidade, distribuição dos poros, saturação em água, saturação irredutível e permeabilidade do meio poroso. O tempo $T_{2}$ está associado às propriedades magnéticas dos prótons, suas interações com outros prótons e moléculas adjacentes. $O$ processo de relaxação magnética transversal que determina a constante $T_{2}$ envolve três mecanismos: relaxação transversal intrínseca (ou bulk) do fluído $\left(T_{2 b u l k}\right)$, a relaxação associada ao contato do fluido com a superfície do grão da rocha $\left(T_{2 \text { sup }}\right)$ e a relaxação associada à difusão molecular $\left(T_{2 \text { dif }}\right)$. Matematicamente, $T_{2}$ pode ser definido como:

$$
\frac{1}{T_{2}}=\frac{1}{T_{2 b u l k}}+\frac{1}{T_{2 s u p}}+\frac{1}{T_{2 d i f}} .
$$

Para soluções salinas, o principal mecanismo de relaxação magnética consiste na relaxação superficial. Para hidrocarbonetos pesados, predomina o componente $T_{2 b u l k}$ e para gás, a relaxação magnética induzida por difusão $T_{2 \text { dif. }}$. Considerando que nessa pesquisa a amostra de rocha foi saturada com solução salina, a relaxação magnética superficial consistiria no fenômeno de relaxação magnética predominante. A constante de relaxação magnética superficial é definida como:

$$
\frac{1}{T_{2 \text { sup }}}=\rho_{2} \frac{S}{V}
$$

onde: $\rho_{2}$ é a relaxividade transversal, $S$ é área da superfície do poro e $V$ é volume do fluido. A razão $\frac{S}{V}$ fornece informação sobre o tamanho do poro, assim, essa expressão (2) explicita a relação existente entre a constante de relaxação transversal $T_{2}$ e o tamanho dos poros na amostra.

Para uma amostra saturada com solução salina, o decaimento da magnetização em função do tempo é dado pela seguinte expressão, considerando um conjunto de poros:

$$
M(t)=\sum_{i=1}^{m} M_{0 i} e^{-\rho_{2}\left(\frac{S}{V}\right)_{i} t} .
$$

De acordo com a expressão (3), quanto menor a razão $\frac{S}{V}$ e portanto, maior o volume do poro, maior o valor da magnetização $M(t)$ e por extensão, maior o valor do sinal de RMN medido.

\section{Cálculo de Porosidade}

Para calcular porosidade a partir da RMN em laboratório, primeiro é necessário calibrar a ferramenta para um volume do fluido correspondente ao volume da amostra. O sinal registrado na ferramenta para o volume da solução salina corresponderá a porosidade de $100 \%$. Em seguida, obtém-se a curva de distribuição $T_{2}$ para a amostra e o valor percentual dessa área $\left(A_{\text {amostra }}\right)$ em relação à magnitude do sinal associado ao volume de fluido de referência $\left(A_{v o l . e q}\right.$, correspondente a porosidade de 100\%) consistirá na medida de porosidade definida por:

$$
\phi_{R M N}=\frac{A_{\text {amostra }}}{A_{\text {vol.eq }}} 100 \%
$$

\section{Índices de fluido livre e de fluido ligado}

É possível fazer inferências sobre o percentual do volume poroso associado ao fluido livre e ao chamado fluido ligado, associado a micro-poros. A partir da definição do chamado Tempo de relaxação magnética transversal de corte $T_{2 \text { corte }}$, que consiste no tempo que divide a curva de distribuição de $T_{2}$ em duas partes, tempos a esquerda do $T_{2 \text { corte }}$ são associados aos fluidos retidos por pressões moleculares e/ou capilares e os tempos à direita do $T_{2 \text { corte }}$ são referentes aos poros nos quais o fluido tem liberdade para se deslocar (Figura 4). A partir disso calcula-se 0 Índice de fluido livre (FFI, do inglês Free Fluid Index) e o Índice de fluido ligado (BVI, do inglês Bulk Volume Irreducible).

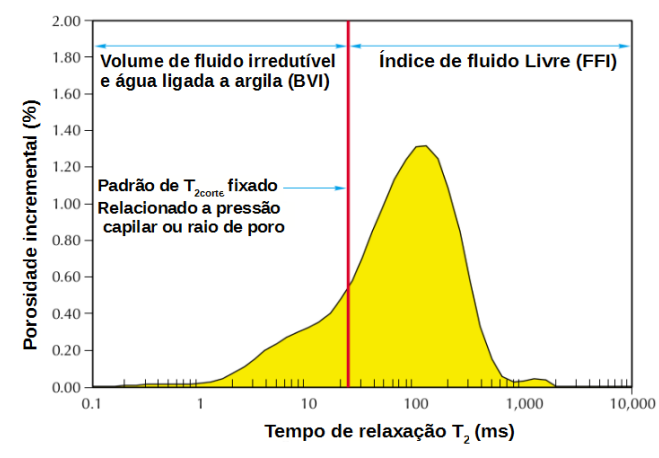

Figure 4: Determinação dos Índices de fluido livre e fluido ligado a partir do T 2corte. Fonte: Coates et al. (1999).

Desse modo, os índices BVI podem ser calculados a partir de:

$$
B V I=\frac{A_{\text {esq }}}{A_{\text {amostra }}} 100 \%,
$$

e como

$$
B V I+F F I=100 \%
$$

teremos que:

$$
F F I=100 \%-B V I
$$


Valores de $T_{2 \text { corte }}$ comuns para rochas clásticas são em torno de $33 \mathrm{~ms}$ e para carbonatos, aproximadamente 90 ms.

O fluido retido por pressões capilares compreende também o espaço poroso associado aos argilominerais. Os argilominerais obstruem poros e assim, contribui para redução de permeabilidade. Para estimar o índice de fluido ligado dos argilominerais é necessário utilizar o $T_{2}$ teórico estabelecido na literatura como o tempo de corte para micro-poros associados à argila: $3 \mathrm{~ms}$ (Coates et al., 1999). A equação (8) explicita o índice de fluido retido por pressões capilares $(B V I)$ em função da parcela associada aos micro-poros de argila $\left(B V I_{\text {argila }}\right)$ e outros micro-poros $\left(B V I^{*}\right)$ :

$$
B V I=B V I_{\text {argila }}+B V I^{*}
$$

\section{Permeabilidade}

A permeabilidade tem relação direta com o tamanho das gargantas dos poros e não com o tamanho dos poros. A partir da RMN é possível estimar valores de permeabilidade em virtude de existir correlação entre o tamanho de garganta do poro e o tamanho do poro. Dois métodos são comumente utilizados para estimar permeabilidade a partir de dados de RMN: o modelo Timur-Coates e o modelo de $T_{2 \text { medio }}$.

\section{Modelo Timur-Coates}

A equação de Timur-Coates estima valores de permeabilidade a partir da relação entre porosidade, índice de fluido livre (FFI) e índice de fluido ligado $(\mathrm{BVI})$ :

$$
\kappa_{c}=\left(\frac{\phi_{R M N}}{c^{\prime}}\right)^{a^{\prime}}\left(\frac{F F I}{B V I}\right)^{b^{\prime}} .
$$

As constantes $a^{\prime}, b^{\prime}$ e $c^{\prime}$ são empíricas e dependem da litologia da amostra. Valores comuns para essas constantes são $a^{\prime}=4, b^{\prime}=2$ e $c^{\prime}=10(m D)^{-1}$ (Gómez et al., 2007; Mao et al., 2013). A unidade de permeabilidade é milidarcy $(m D)$.

\section{Modelo de $T_{2}$ médio}

A permeabilidade estimada a partir desse modelo é função da porosidade e da média geométrica dos valores de $T_{2}\left(T_{2 G M}\right)$ :

$$
\kappa_{\text {medio }}=c^{\prime} \phi^{a^{\prime}} T_{2 G M}^{b^{\prime}} .
$$

Novamente, $a^{\prime}, b^{\prime}$ e $c^{\prime}$ são constantes empíricas cujos valores dependem da litologia da rocha analisada. Valores comumente atribuídos a tais constantes são, respectivamente: 4, 2 e 4,5mD/ms ${ }^{2}$ (Daigle e Dugan, 2009; Mao et al., 2013).

\section{Cálculo de porosidade a partir de Ultrapicnômetro}

O cálculo de porosidade de amostra de rochas via Ultrapicnômetro é um método de obtenção de porosidade bastante comum na petrofísica. O Ultrapycnometer 1000 , fabricado pela Quantachrome, foi utilizado nessa pesquisa. Nesse Ultrapicnômetro, o princípio físico para medida do volume não poroso $\left(\Delta V_{N P}\right)$ das amostras consiste na injeção de gás hélio. $O$ volume de espaços vazios $\left(\Delta V_{v}\right)$ é definido como:

$$
\Delta V_{v}=\Delta V_{t}-\Delta V_{N P}
$$

onde $\Delta V_{t}$ é volume total da amostra. A partir das medidas no Ultrapicnômetro, a porosidade foi calculada pela substituição da expressão 11 na expressão 12.

$$
\phi_{t}=\frac{\Delta V_{v}}{\Delta V_{t}} 100 \%
$$

\section{Resultados e Discussões}

Das 31 amostras analisadas nesse trabalho, 13 amostras são associadas à localidade de Ponta de Nossa Senhora (Amostras PNS) e 18 amostras são oriundas dos afloramentos em Loreto (Amostras LOR). Todas as amostras foram analisadas no que concerne à porosidade a partir da RMN e do Ultrapicnômetro. A Figura 5 apresenta a distribuição dos valores de porosidade calculados a partir do Ultrapicnômetro e da RMN, bem como, o coeficiente de correlação entre eles $(R \approx 0,02)$. Os valores de porosidade obtidos com o Ultrapicnômetro foram maiores do que aqueles calculados a partir da RMN. A baixa correlação entre os dois métodos de medida de porosidade das amostras pode ser explicado pela diferença no princípio físico de cada método: para obter a porosidade a partir da RMN, as amostras são saturadas com uma solução salina, enquanto que, no Ultrapicnômetro, gás Hélio é injetado no espaço poroso. Como a molécula de gás Hélio é menor do que a molécula de água, a primeira tem maior capacidade de penetração em micro-poros, por isso, a porosidade calculada a partir das medidas no Ultrapicnômetro seria representativa da porosidade total das amostras.

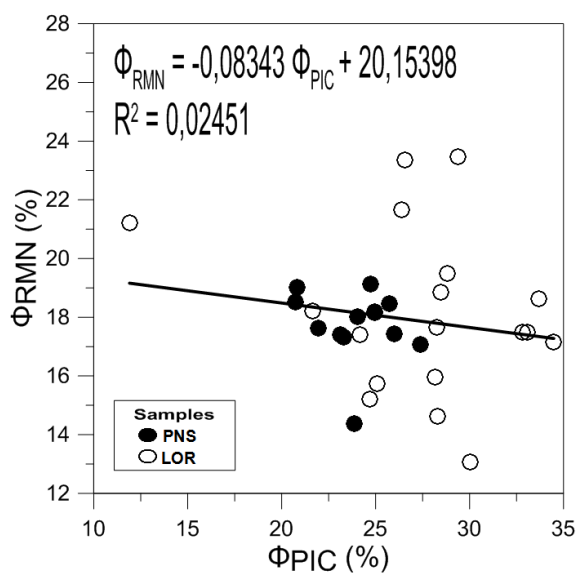

Figure 5: Correlação entre a porosidade calculada a partir do método da RMN e a porosidade obtida a partir do Ultrapicnômetro.

Os valores médios de porosidades obtidos a partir do Ultrapicnômetro e da RMN para as amostras da localidade 
de Ponta de Nossa Senhora são, respectivamente: $23,97 \%, 17,74 \%$. Enquanto que, para as amostras da localidade de Loreto, os valores dessas porosidades foram, respectivamente: $27,56 \%$ e $18,15 \%$. Como pode ser observado, os valores de porosidades obtidos para as amostras de prefixo LOR em ambos métodos são maiores do que aqueles calculados para as amostras de prefixo PNS. Além disso, conforme classificação apresentada por Rosa et al.(2006), rochas com esses valores de porosidade são classificadas como rochas de porosidade intermediária a muito boa.

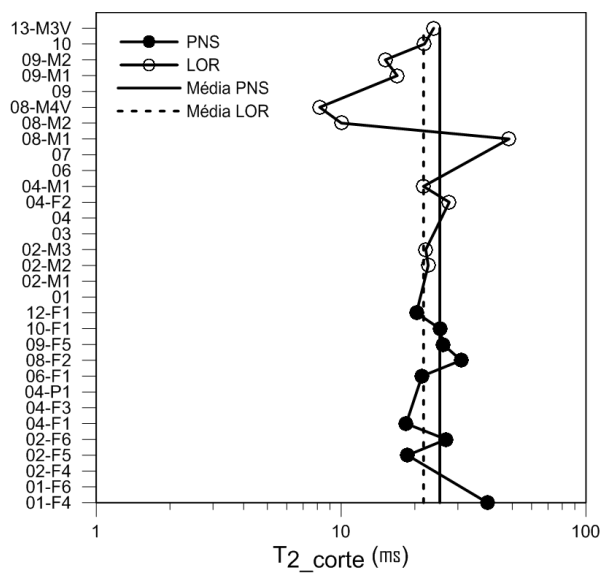

Figure 6: Distribuição dos valores de $T_{2 \text { corte }}$ em função das amostras, bem como, valores médios associados às amostras LOR e PNS.

Para análise dos índices de fluido livre e índice de fluido retido por pressão capilar (ou fluido ligado ou irredutível), bem como estimativas de permeabilidade, as amostras foram submetidas por uma segunda vez ao processo de saturação para serem colocadas sob pressão no prato poroso e assim, alcançarem a condição de saturação irredutível. O processo de saturação favorece a dissolução de constituintes friáveis e algumas amostras sofreram degradação: alteração significativa na massa ou aparecimento de fraturas. Assim, a segunda saturação, associada à pressão a qual as amostras foram submetidas no prato poroso, implicou na redução do número de amostras que foram analisadas quanto à permeabilidade e aos índices de fluido livre e ligado. Após uma segunda saturação, das 31 amostras, somente foi possível realizar medidas no equipamento de RMN em 20 amostras: 9 amostras de prefixo PNS e 11 amostras são LOR.

Com as curvas de distribuição dos tempos de relaxação magnética transversal $\left(T_{2}\right)$ para a amostra considerada $100 \%$ saturada e em condição de saturação irredutível, o $T_{2 \text { corte }}$ para cada uma das 20 amostras foi determinado. A Figura 6 apresenta a distribuição do $T_{2 \text { corte }}$ em função das amostras, bem como, os valores médios desse tempo para as amostras dos grupos LOR e PNS. Os valores médios para as amostras do grupo LOR e PNS são: $21,71 \mathrm{~ms}$ e $25,29 \mathrm{~ms}$, respectivamente. Vale destacar que apesar das dificuldades associadas à determinação das condições de rocha $100 \%$ saturada e em saturação irredutível, os valores médios de $T_{2 \text { corte }}$ obtidos a partir das medidas laboratoriais estão próximos do valor médio associado na literatura para arenitos (33ms).

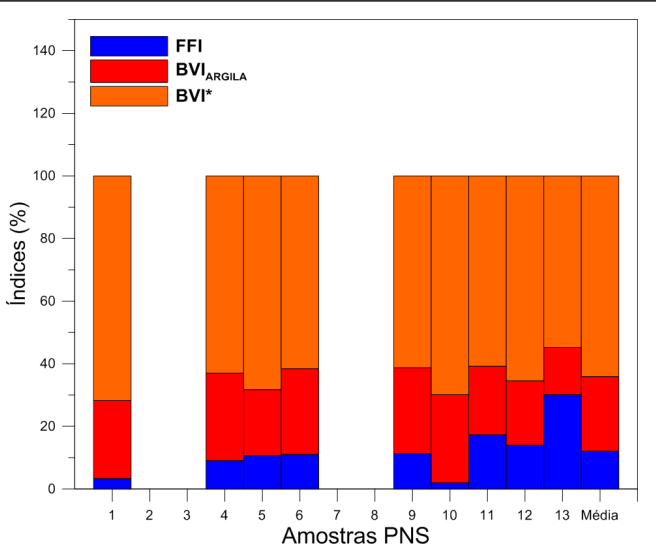

(a)

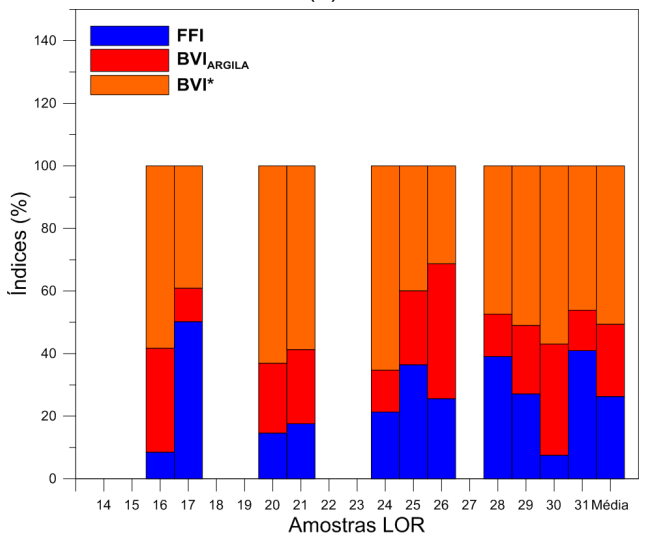

(b)

Figure 7: Distribuição dos índices de fluido livre (FFI), fluido ligado aos micro-poros de argila $\left(B V I_{\text {argila }}\right)$ e fluido ligado a outros micro-poros $\left(B V I^{*}\right)$ para (a) amostras PNS e (b) amostras LOR. A última coluna de cada gráfico apresenta os valores médios desses índices para cada grupo de amostras.

Uma vez definido o $T_{2 \text { corte }}$ das amostras, os índices de fluido livre (FFI), fluido ligado aos micro-poros de argila $\left(B V I_{\text {argila }}\right)$ e fluido ligado a outros micro-poros $\left(B V I^{*}\right)$ foram calculados. A Figura 7 apresenta a distribuição desses índices para as amostras PNS e LOR, bem como, os valores médios dos índices para cada grupo de amostras. Os valores médios dos índices $\mathrm{FFI}, B V I_{\text {argila }} \mathrm{e}$ $B V I^{*}$ das amostras oriundas da localidade de Ponta de Nossa Senhora são, respectivamente: $12,08 \%, 23,83 \%$ e $64,09 \%$. Os valores desses índices para as amostras da localidade de Loreto são, respectivamente: $26,27 \%$, $23,09 \%$ e $50,64 \%$.

O grupo de amostras PNS apresenta os valores médios dos índices de fluido ligado a micro-poros de argila e outros micro-poros maiores do que o grupo LOR. O grupo LOR apresenta o valor médio do índice de fluido livre correspondente a mais do que o dobro do valor médio desse índice para as amostras PNS, o que indica que a porosidade efetiva das amostras de prefixo LOR é maior do que a porosidade efetiva das rochas de prefixo PNS.

A estimativa de permeabilidade a partir do modelo TimurCoates utiliza no cálculo os valores dos índices de fluido livre e fluido retido por pressões capilares. Os 
valores médios de permeabilidade obtidos a partir desse modelo associados às amostras dos grupos LOR e PNS foram, respectivamente: $1,49 \mathrm{mD}$ e $0,31 \mathrm{mD}$. Já os valores médios de permeabilidade obtidos a partir da utilização do modelo do $T_{2}$ médio $\left(T_{2 G M}\right)$, foram, respectivamente: $0,48 \mathrm{mD}$ e $0,30 \mathrm{mD}$. A Figura 8 apresenta a distribuição das permeabilidades estimadas a partir dos dois métodos, bem como, o coeficiente de correlação entre elas. Apesar das diferenças referentes aos modelos de estimativa de permeabilidade, os resultados sinalizam que as amostras LOR tendem a apresentar valores de permeabilidade maiores do que as rochas do grupo PNS.

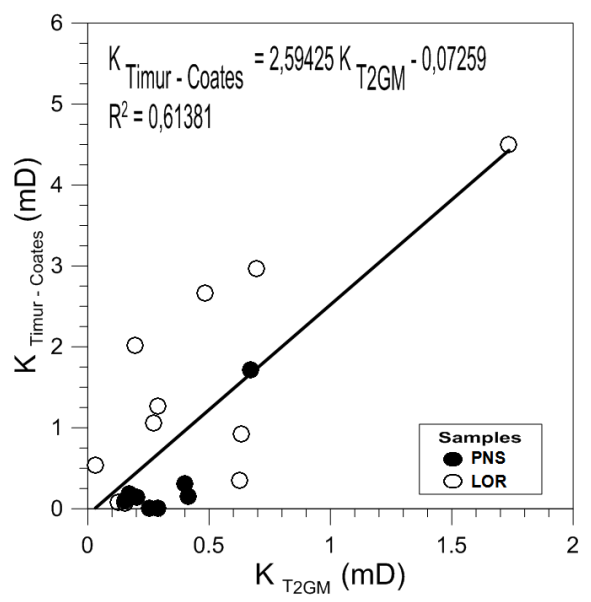

Figure 8: Correlação entre permeabilidades estimadas a partir do modelo Timur-Coates e do modelo de $T_{2 G M}$.

\section{Conclusões}

A análise laboratorial das 31 amostras de arenitos da Formação Maracangalha, oriundas de afloramentos em Loreto e Ponta de Nossa Senhora na Ilha dos Frades a partir do equipamento de Ressonância Magnética Nuclear (RMN) viabilizaram que tais amostras fossem caracterizadas em termos de porosidade, índice de fluidos livre (FFI), índice de fluido retido por pressão capilar (BVI), bem como, estimativas de permeabilidades.

Os resultados obtidos evidenciaram como os arenitos da Formação Maracangalha das duas localidades da llha dos Frades são heterogêneos. Os arenitos do grupo LOR destacam-se como as amostras analisadas que apresentam os maiores valores de porosidade, índice de fluido livre e permeabilidade. Enquanto que, os arenitos do grupo PNS apresentam os maiores valores associados aos indices de fluido retido por pressão capilar.

A natureza mais argilosa das amostras PNS podem explicar a menor porosidade e a baixa permeabilidade desse grupo de rochas. Os valores de porosidade, permeabilidade e índice de fluido livre associados às amostras do afloramento de LOR indicam que tais amostras tendem a constituir reservatórios com maior capacidade de armazenamento, transmissão e recuperação de hidrocarbonetos. As características petrofísicas dos arenitos de Loreto sugerem que tais amostras são representativas do Membro Caruaçu, o principal reservatório de hidrocarbonetos da Formação Maracangalha.

\section{Referências}

Bittencourt, A. C. D. S. P.; Ferreira, Y. D. A.; Di Napoli, E. Alguns aspectos da sedimentação na Baía de Todos os Santos, Bahia. Revista Brasileira de Geociências, v. 6, n. 4, 246-263, 2018.

Caixeta, J. M. (1988) Estudo faciologico e características de reservatorio dos arenitos produtores de gas do campo de jacuipe (cretaceo inferior), Bacia do Reconcavo, Brasil [Unpublished M. Sc. thesis]: University Federal de Ouro Preto, Ouro Preto, Brazil.

Coates, G. R.; Xiao, L.; Prammer, M. G. et al. (1999) NMR logging: principles and applications, vol. 234, Haliburton Energy Services Houston.

Daigles, H.; Dugan, B. Extending NMR data for permeability estimation in fine-grained sediments. Marine and Petroleum Geology, v. 26, n. 8, 1419-1427, 2009.

Gómez, H., Stinco, L. P., Nawratil, A. E., Lopetrone, J., Romero, P. A., e Saavedra, B. E. Correction of bound and free-fluid volumes in the timur-coates permeability equation for the presence of heavy oil: a case study from the golfo san Jorge Basin, Argentina. In Latin American e Caribbean Petroleum Engineering Conference. Society of Petroleum Engineers, 2007.

Kenyon, W.E. Petrophysical Principles of Applications of NMR Logging, The Log Analyst 38, 21-43, 1997.

Magalhaes, A. J. C. Modelo deposicional e características de reservatório dos arenitos das Camadas Caruaçu (Cretáceo inferior) no Campo de Miranga Profundo, Bacia do Recôncavo, Brasil. Dissertação de Mestrado. Universidade Federal de Ouro Preto, Ouro Preto-MG, 1990.

Magnavita, L. P.; Silva, R. R. da; Sanches, C. P. Guia de campo da Bacia do Recôncavo, NE do Brasil. Boletim de Geociências da PETROBRAS, v. 13, n. 2, 301-334, 2005.

Mao, Z. et al. Estimation of permeability by integrating nuclear magnetic resonance (NMR) logs with mercury injection capillary pressure (MICP) data in tight gas sands. Applied Magnetic Resonance, v. 44, n. 4, 449-468, 2013.

Martinez, G. A.; Lorne A. D. Petrophysical measurements on shales using NMR. SPE/AAPG Western Regional Meeting. Society of Petroleum Engineers, 2000.

Medeiros, V. C. D. Geologia e recursos minerais da folha Sousa SB. 24-ZA. 2008.

Milhomem, P. S.; Maman, E. J.; Oliveira, F. M.; Carvalho, M. S. S. e Lima, W. S. (2003).Bacias sedimentares brasileiras: Bacia do recôncavo, Fundação Paleontológica Phoenix, 5(51).

Rosa, A. J. et al. Engenharia de reservatórios de petróleo. Interciência, 2006.

\section{Agradecimentos}

Esta pesquisa faz parte da Rede de Pesquisa e Desenvolvimento de Gás Não Convencional no Brasil - GASBRAS. Agradecemos ao INCT-GP, FINEP, CNPq, CAPES e FAPESB, que nos deram o suporte financeiro e os recursos necessários para o desenvolvimento dessa pesquisa. 